\title{
Desenvolvimento e Propriedades Psicométricas do Teste Informatizado de Avaliação das Funções Executivas
}

\author{
Glauce Karine Conti de Freitas Elage ${ }^{1}$ (D), Alessandra Gotuzo Seabra (D) \\ Universidade Presbiteriana Mackenzie, São Paulo-SP, Brasil
}

\section{RESUMO}

Funções executivas (FE) são habilidades que permitem o autocontrole comportamental e cognitivo e estão relacionadas a diversos desfechos ao longo da vida. O uso de testes informatizados para avaliar as FE pode facilitar a precisão dos registros, a padronização e a análise dos dados. Este estudo objetivou desenvolver um instrumento informatizado para avaliar FE em crianças de 4 a 10 anos e analisar características psicométricas. Foram conduzidas cinco etapas: 1. Definição teórica e metodológica; 2. Construção dos itens; 3 . Estudo piloto; 4. Análise de juízes; e 5. Estudos psicométricos de validade e fidedignidade. As tarefas informatizadas mostraram-se adequadas para o público-alvo, conforme avaliação dos juízes. As diferentes tarefas de memória de trabalho, inibição e flexibilidade cognitiva apresentaram correlações significativas entre si e a maioria das medidas no teste-reteste evidenciou estabilidade na mensuração. Portanto, os resultados sugerem viabilidade para uso do instrumento no contexto brasileiro.

Palavras-chave: funcionamento executivo; infância; avaliação informatizada; psicometria.

ABSTRACT - Development and psychometric properties of the Computerized Executive Functions Assessment Test Executive functions (EF) are skills linked to behavioral and cognitive self-control and are related to various outcomes throughout life. The use of computerized tests to evaluate EFs can facilitate the accuracy of records, standardization and data analysis. This study aimed to develop a computerized instrument for the EF assessment of children aged 4 to 10 years, and to seek psychometric evidence. Five steps were carried out: 1 . Theoretical and methodological definition; 2 . Construction of the items; 3 . Pilot study; 4 . Analysis of experts; and 5. Psychometric studies of validity and reliability. The computerized tasks proved to be suitable for the target audience according to the expert's evaluation. The results between the different tasks of working memory, inhibition and cognitive flexibility showed significant correlations and most test-retest measures showed stability in the measurement. Therefore, the results indicate the feasibility of using the instrument in the Brazilian context.

Keywords: executive functioning; childhood; computerized assessment; psychometry.

RESUMEN - Desarrollo y propiedades psicométricas del Test Informatizado de Evaluación de las Funciones Ejecutivas Funciones ejecutivas (FE) son habilidades que permiten el autocontrol conductual y cognitivo y están relacionadas con diversos resultados a lo largo de la vida. El uso de tests informatizados para evaluar las FE puede facilitar la precisión de los registros, la estandarización y el análisis de datos. Este estudio tuvo como objetivo desarrollar un instrumento informatizado para la FE para niños de 4 a 10 años, y analizar evidencias psicométricas. Fueron ejecutados cinco pasos: 1. Definición teórica y metodológica; 2. Construcción de los ítems; 3. Estudio piloto; 4. Análisis de jueces; y 5. Estudios psicométricos de validez y fiabilidad. Las tareas informatizadas demostraron ser adecuadas para el público objetivo según la evaluación de los jueces. Las diferentes tareas de memoria de trabajo, inhibición y flexibilidad cognitiva mostraron correlaciones significativas entre sí y la mayoría de las medidas test-retest presentaron estabilidad en la medición. Por lo tanto, los resultados sugieren la viabilidad del instrumento para el contexto brasileño.

Palabras clave: funcionamiento ejecutivo; infância; evaluación informatizada; psicometria.

Funções executivas (FE) são habilidades cognitivas envolvidas no controle e direcionamento de um comportamento a uma meta específica. Podem ser definidas como um sistema de supervisão importante para a organização de uma sequência de ações. As FE, de acordo com o modelo teórico sumarizado por Diamond (2013) a partir de pesquisas prévias (como Miyake \& Friedman, 2012; Miyake et al., 2000), estão vinculadas a habilidades específicas, tais como a memória de trabalho, que consiste na capacidade de armazenamento e atualização da informação enquanto realiza alguma operação; o controle inibitório, que se refere à capacidade de inibir uma resposta prepotente ou automatizada para a conclusão adequada de uma tarefa; e, ainda, a flexibilidade cognitiva, definida como a habilidade de flexibilizar o foco de atenção entre diferentes demandas.

As habilidades das FE desenvolvem-se ao longo da vida, podendo atingir a maturidade somente no início da vida adulta (Huizinga et al., 2006). Esse período prolongado se justifica pelas constantes mudanças

1 Endereço para correspondência: Rua Iaciara, Quadra B - 02, Lote 10, 74884-573, Goiânia, GO. Tel.: (62) 98185-8866. E-mail: glauceconti@gmail.com Artigo derivado da 'Dissertação de mestrado' de 'Glauce Karine Conti de Freitas Elage' com orientação de 'Alessandra Gotuzo Seabra', defendida em '2016' no programa de pós-graduação 'Distúrbios do desenvolvimento' da 'Universidade Presbiteriana Mackenzie. 
neuroanatômicas e bioquímicas ocorridas ao longo do desenvolvimento, como o aumento da extensão dentrítica, do número de receptores de dopamina e da mielinização prolongada do córtex pré-frontal (Diamond, 2001). Contudo, embora haja uma longa trajetória, o início do desenvolvimento ocorre em fases bastante precoces. De fato, estudos que buscaram investigar a cognição em bebês apontaram que, antes do primeiro ano de vida, já é possível observar habilidades para operar informações armazenadas (Blankenship et al., 2019; Johansson et al., 2014) e indícios de regulação intencional do comportamento (Rothbart et al., 2011).

No período pré-escolar, entre 3 e 6 anos de idade, ocorrem avanços rápidos, porém não homogêneos, no desenvolvimento das FE (Best et al., 2011; Bierman \& Torres, 2016). Habilidades como a flexibilidade cognitiva tornam-se mais evidentes. Crianças por volta dos 3 anos conseguem solucionar tarefas simples de flexibilidade, porém o sucesso em tarefa mais complexas, que envolvem duas ou mais informações, ocorre por volta dos 4 ou 5 anos (Zelazo et al., 2003). A capacidades de postergar gratificação e manipular maior número de informações em mente também se mostram mais eficientes nesse período (Dawson \& Guare, 2018; Duckworth et al., 2019).

Nos anos seguintes, no período escolar, saltos na maturação em regiões frontais permitem que as FE atinjam níveis de funcionamento próximos aos de um adulto (Diamond, 2001). Consequentemente, as crianças tornam-se mais capazes de se envolver em situações complexas que exigem abstração, autorregulação, manejo de situações de estresse, inibição de comportamentos indesejáveis e distratores, favorecendo o desenvolvimento das habilidades acadêmicas (Wolf \& McCoy, 2019) e o progresso nas experiências sociais, como a melhora na habilidade de modificar e adaptar um comportamento mediante uma pista social (Christ et al., 2017; McClelland et al., 2014).

Há evidências de que, com o desenvolvimento, os componentes das FE passam de uma estrutura mais unitária para uma em que eles se tornam mais específicos e distintos entre si, apesar de ainda haver divergências sobre a idade e a hierarquia de desenvolvimento desses componentes (Huizinga et al., 2006; Miyake \& Friedman, 2012). Fatores biológicos, como prematuridade e estresse tóxico na gestação; ambientais, como a negligência e a violência; e sociais, como o nível socioeconômico e cultural, podem impactar negativamente o desenvolvimento das FE ao longo da vida (Center on the Developing Child at Harvard University, 2011). Por sua vez, baixos desempenhos em FE estão presentes em diversas condições clínicas, como Transtorno de Déficit de Atenção (TDAH) (Barkley, 2011), Transtornos do Espectro Autista (TEA) (Merchán-Naranjo et al., 2016) e Transtorno de Aprendizagem (Bausela-Herreras et al., 2019), o que corrobora a importância da avaliação dessas funções em idades precoces, preferencialmente antes do ingresso no ensino fundamental ou durante seus primeiros anos.

Nas últimas décadas, extensas pesquisas foram conduzidas acerca da relevância das FE sobre diversos domínios, como raciocínio, compreensão e leitura (Baggetta \& Alexander, 2016) e, consequentemente, houve um maior interesse por instrumentos e estratégias de investigações das FE. No Brasil, Carreiro et al. (2014) e Seabra et al. (2017) realizaram revisões a fim de traçar um panorama dos instrumentos de avaliação de FE no Brasil. Os dados fornecidos pelos dois estudos indicam quantidade limitada de instrumentos disponíveis para avaliação das FE de crianças, sendo que alguns dos instrumentos apontados, apesar de possuírem demanda sobre FE, foram desenvolvidos para avaliar outras habilidades, tais como o Teste de Fluência Verbal (Fonseca et al., 2016), originalmente desenvolvido para avaliar processos linguísticos; o teste de Figuras Complexas de Rey (Oliveira \& Rigoni, 2010), utilizado para investigar processos visuoconstrutivos, e a Escala Wechsler de Inteligência para Criança - WISC-IV (Rueda et al., 2013) que, embora permita a avaliação das FE por meio de alguns subtestes, é um instrumento para avaliação da inteligência. Adicionalmente, de acordo com as revisões, nenhum dos instrumentos fornecem medidas separadas para as três habilidades básicas das FE, a saber: memória de trabalho, controle inibitório e flexibilidade cognitiva, conforme modelo de Diamond (2013).

Quando se refere ao uso de instrumentos informatizados para avaliar as FE, disponíveis no Brasil e com propriedades psicométricas adequadas, o número ainda é mais restrito, apesar de, internacionalmente, muitos pesquisadores se interessarem pela informatização de testes psicológicos, elaboração de plataformas, adaptações informatizadas de versões tradicionais e criação de software, como o Cambridge Neuropsychologic al Test Automated Battery (CANTAB) que, dentre outros domínios, avalia as FE (Olea et al., 2010; Parsons, 2016).

No Brasil, esse interesse ocorreu mais tardiamente, com um crescente número de pesquisas na área, mas avanços ainda lentos quando comparados a países desenvolvidos (Joly et al., 2005). Em relação à avaliação das FE, há uma versão computadorizada da tarefa Children's Gambling Task (CGT-Br), que avalia a tomada de decisão em crianças entre 3 e 5 anos, desenvolvida e com estudos psicométricos publicados (Mata et al., 2013), porém indisponível para uso clínico; uma adaptação brasileira do instrumento de Avaliação Automatizada da Memória Operacional (Automated Working Memory Assessment AWMA) (Santos \& Engel, 2009) desenvolvido para investigar, dentre outros domínios, a memória de trabalho de crianças a partir de 5 anos; o Teste de Fluência Verbal computadorizado, que avalia crianças de 6 a 14 anos, não está disponível para uso, contudo apresenta estudos psicométricos (Dias et al., 2013); o teste de Stroop computadorizado (Dias et al., 2013), que investiga o controle 
inibitório de crianças 10 a 16, embora com propriedades psicométricas publicadas não está disponível para uso; e, mais recentemente, o Jogo das Cartas Mágicas, desenvolvido por Uehara et al. (2016), que objetiva avaliar as FE. Estudos psicométricos foram desenvolvidos, mas o instrumento também não está disponível para uso. Plataformas para avaliação de FE têm sido desenvolvidas, como a EF Solutions, que contém algumas tarefas informatizadas de FE (Malloy-Diniz et al., 2020).

A avaliação das FE a partir de instrumentos informatizados apresenta diversas vantagens, como a confiabilidade na aplicação, padronização das instruções, melhor controle do tempo, bem como a eficiência da correção e conversão dos escores, possibilitando ainda a utilização de diferentes estímulos de multimídia (Parson, 2016; Primi, 2010). Nesse contexto, diante da importância das FE para diversos desfechos ao longo da vida, das evidências de sua estrutura multifatorial e das vantagens das avaliações informatizadas, este estudo teve como objetivo principal a construção de um instrumento informatizado para avaliar FE em crianças de 4 a 10 anos de idade. Objetivou-se desenvolver um instrumento com tarefas lúdicas, atrativas para as crianças, com instruções detalhadas, padronizadas e com correção automática para avaliar as FE de crianças e ainda que proporcione condições de investigar a organização tríplice das FE (controle inibitório, memória de trabalho e flexibilidade cognitiva), visto que o presente artigo relata as etapas do desenvolvimento do instrumento, bem como os estudos que investigaram a precisão das medidas e evidências de validade do instrumento.

\section{Método}

O processo de desenvolvimento do instrumento fundamentou-se na teoria clássica de construção de um instrumento de medida, seguindo os parâmetros e procedimentos rígidos do tipo teórico, empírico e analítico, e respaldou-se nos conceitos teóricos da neuropsicologia acerca das FE, conforme descrito nas seções seguintes. Para a execução dos procedimentos, dividiu-se o estudo em cinco etapas: 1. definição teórica e metodológica; 2. construção dos itens e desenvolvimento da versão preliminar; 3. estudo piloto; 4. análise dos juízes; e 5. estudos psicométricos de validade e fidedignidade. $\mathrm{O}$ projeto foi submetido e aprovado pelo Comitê de ética da instituição responsável. Os responsáveis pelas escolas e pelas crianças participantes forneceram a autorização para participação na pesquisa, além do assentimento das próprias crianças.

\section{Etapa 1 - Definição Teórica e Metodológica}

A primeira etapa do trabalho envolveu um processo de busca à literatura no que concerne aos modelos teóricos sobre FE e aos instrumentos e tarefas de avaliação dessas funções de uso frequente na prática clínica em contexto nacional e internacional. Foram usadas como base as diretrizes do International Test Commission - ITC (2010). Como substrato teórico, optou-se pela utilização do modelo descrito por Diamond (2013) que organiza as FE nas três funções básicas de memória de trabalho, controle inibitório e flexibilidade cognitiva, conforme já descrito na Introdução.

A partir de ideias derivadas das atividades selecionadas na literatura e, a fim de torná-las mais atraentes para a população infantil, optou-se por planejar atividades de caráter lúdico. Para tanto, foram selecionadas algumas personagens para inclusão nas tarefas. Para a escolha das personagens, foram realizadas, inicialmente, buscas nos bancos de imagens disponíveis na internet e oito imagens foram selecionadas. Tais imagens foram apresentadas a 23 profissionais que trabalham com crianças na área da saúde e educação para que escolhessem, entre as oito imagens, quatro que julgassem mais atraentes para crianças de 4 a 10 anos de idade e que pudessem promover a identificação independentemente de gênero.

\section{Etapa 2 - A Construção dos Itens e o Desenvolvimento da Versão Preliminar}

Após a definição do tipo de tarefa, do funcionamento e da escolha das personagens, as atividades foram desenvolvidas, primeiramente no papel, incluindo todas as fases de treinos e de testes. Após estruturar a ordem e o formato, as tarefas foram encaminhadas para equipe de tecnologia e desenvolvimento de software que criou um protótipo informatizado da versão de papel apresentada, utilizando para isso a ferramenta XCode da Apple e a linguagem Objective-C.

\section{Etapa 3 - Estudo Piloto e Desenvolvimento das Tarefas Informatizadas}

A versão informatizada desenvolvida preliminarmente foi aplicada em 10 crianças com idade entre 4 e 10 anos, da cidade de Goiânia, sendo uma criança de 4 anos, uma de 5 , duas de 6 , duas de 7 , duas de 8 , uma de 9 e uma de 10 anos, todas de escolas privadas. O objetivo do estudo piloto foi investigar a compreensão das instruções, por parte das crianças; avaliar o tempo despendido em cada tarefa e avaliar o interesse das crianças pelas tarefas. Após a aplicação, os resultados foram discutidos entre as autoras do teste e as modificações necessárias foram encaminhadas para a equipe responsável em desenvolver as tarefas computadorizadas. Assim, criou-se uma versão para ser submetida à análise de conteúdo por parte de juízes.

\section{Etapa 4 - A Análise dos Juízes}

Para a construção de um instrumento de avaliação, recomenda-se que submeta o instrumento a uma amostra populacional (juízes), considerada especialista na área, para realizar adequado julgamento dos itens do instrumento (Urbina, 2007). Para isso, participaram dessa etapa do estudo dois doutores, um em Psicologia e um em 
Distúrbios do Desenvolvimento, ambos especialistas em Neuropsicologia e que atuam tanto na área clínica quanto na acadêmica. A análise foi realizada por meio de um questionário com 46 questões (fechadas e abertas), que investigaram a definição e a coerência dos construtos do teste, a adequação das atividades para o público infantil, o design, o layout, o cadastro, as instruções, os áudios, as fases de treino, as regras de interrupção e as formas de registro dos resultados. Os questionários foram entregues aos juízes, juntamente com um tablet com o aplicativo.

\section{Etapa 5 - Estudos Psicométricos Iniciais}

Para os estudos psicométricos inicias, participaram 51 crianças de 4 a 10 anos de idade, da cidade de Goiânia, sendo 34 meninos e 17 meninas. Todas as crianças foram submetidas ao Teste de Avaliação das Funções Executivas (TAFE). Foi realizada a análise de correlação de Spearman entre as principais medidas das diferentes habilidades avaliadas no TAFE. Para a análise de fidedignidade do instrumento, dez crianças foram novamente submetidas ao TAFE, após três meses da primeira aplicação e foram realizadas correlações de Spearman entre as pontuações do TAFE aplicado nos dois diferentes momentos. Foram usadas correlações não paramétricas devido ao pequeno número amostral e ao fato de algumas medidas não terem revelado normalidade de distribuição.

\section{Resultados}

\section{Resultados das Etapas 1 e 2}

Como resultado das etapas 1 e 2, foram desenvolvidas as tarefas informatizadas que avaliaram três componentes das funções executivas: memória de trabalho, flexibilidade cognitiva e inibição. Cada tarefa informatizada está descrita a seguir, e as imagens de cada uma são apresentadas na Figura 1.
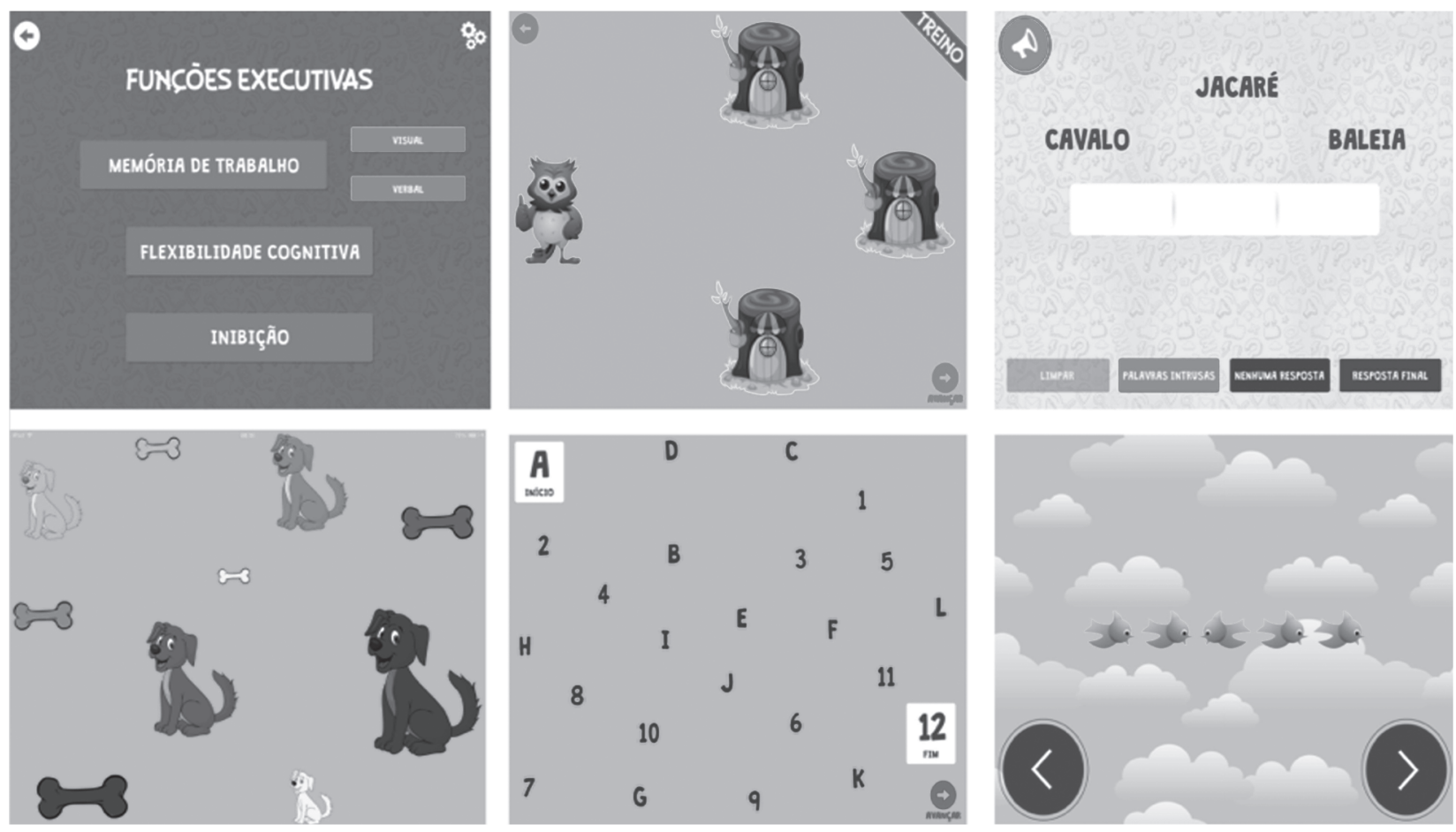

Figura 1. Tarefas do Teste de Avaliação das Funções Executivas

\section{Tarefa de Memória de Trabalho}

O modelo teórico que fundamentou o desenvolvimento dessa tarefa foi o modelo de componentes múltiplos de Baddeley (2000). De acordo com ele, a memória de trabalho é constituída por subsistemas e, conforme a natureza do estímulo (visuoespacial ou verbal), um dos subsistemas assume o processo de arquivamento e de manipulação das informações. Seguindo esse conceito, foram elaboradas duas atividades diferentes para avaliar a memória de trabalho, considerando suas diferentes naturezas: 1. Tarefa de memória de trabalho visuoespacial; e 2. Tarefa de memória de trabalho verbal, descritas a seguir.

\section{Memória de Trabalho Visuoespacial}

A tarefa utilizada como suporte no desenvolvimento da tarefa de memória de trabalho visuoespacial do TAFE foi a Tarefa de Cubos de Corsi (Corsi, 1972), uma tarefa 
clássica amplamente empregada na avaliação da Memória de Trabalho Visuoespacial na clínica neuropsicológica. Ao longo dos anos, várias versões foram criadas, incluindo uma versão computadorizada de Vandierendonk et al. (2004), e estudos psicométricos foram realizados, por exemplo, por Farrell-Pagulayan et al. (2006) e Santos et al. (2005). Todos esses estudos indicaram parâmetros psicométricos adequados, fator que foi determinante para a escolha dessa tarefa. Contudo, para o TAFE, buscou-se desenvolver uma tarefa mais lúdica e atraente para o público infantil. Por esse motivo, os cubos da tarefa clássica foram substituídos por imagens de casas e foi incluído à tarefa uma personagem (uma coruja) que interage com a criança e fornece as instruções para execução da tarefa. A criança é orientada a prestar atenção ao movimento da coruja e, após a interrupção do movimento, indicar com o toque na tela as casas que a coruja visitou.

Antes da criança iniciar o teste, há duas telas de treino para que ela se familiarize com a tarefa e com o toque na tela. Após a finalização do treino, a tarefa é iniciada com grau crescente de dificuldade. O desempenho da criança é registrado de forma automática. Assim, são contabilizados os números de sequências respondidas de forma correta e incorreta. O teste é interrompido se a criança errar três telas consecutivas ou, ainda, se realizar corretamente as 12 telas que compõem o teste. Após a finalização da primeira parte da tarefa (ordem direta), por interrupção ou finalização, surge uma nova tela e a criança é dirigida para a segunda etapa (ordem inversa). Todo o funcionamento da parte inversa ocorre conforme a etapa anterior, com uma única diferença: os movimentos da coruja devem ser repetidos pela criança na ordem inversa à ordem apresentada no modelo. Para indicar à criança a mudança, além da instrução específica para essa etapa, a tela da ordem inversa é apresentada com alteração nas cores dos estímulos.

\section{Memória de Trabalho Verbal}

Para a atividade de memória de trabalho verbal, foram encontradas na literatura as tarefas de Repetição de Dígitos (DigitSpan). Essa é uma tarefa amplamente utilizada e vários estudos de normatização para a população infantil brasileira foram realizados (Figueiredo \& Nascimento, 2007; Santos \& Bueno, 2003), além de estudos de validade e fidedignidade que revelam valores altos de coeficiente de confiabilidade (Williams et al., 2003). No TAFE, os números utilizados como estímulos nas versões citadas foram substituídos por nomes de animais comuns para a população brasileira.

As crianças devem repetir as palavras, primeiramente na ordem direta. Após a finalização da primeira parte da tarefa (ordem direta), por interrupção ou finalização, surge uma nova tela, e a criança é dirigida para a segunda etapa (ordem inversa). São 16 telas para cada etapa, iniciando com duas palavras e finalizando com nove. A quantidade e a sequência de etapas, corretas e incorretas, são contabilizadas. O teste é interrompido se a criança errar três etapas consecutivas ou se concluir as 16 etapas, quando o teste é finalizado.

\section{Tarefa de Flexibilidade Cognitiva}

As atividades de flexibilidade cognitiva foram elaboradas conforme o princípio do clássico Trail Making Test (Partington \& Leiter, 1949). Para o TAFE, a tarefa foi subdividida em duas diferentes atividades: uma tarefa para as crianças em idade pré-escolar, que independe de conhecimentos numérico e alfabético, e uma atividade indicada para as crianças em idade escolar que já tenham conhecimentos numérico e alfabético. Tais atividades são descritas a seguir.

\section{Flexibilidade Cognitiva para Crianças em Idade Pré-escolar}

A atividade elaborada para avaliar a flexibilidade cognitiva em crianças em idade pré-escolar consiste em uma adaptação, para uso em tablet, do Teste de Trilhas para PréEscolares, desenvolvido por Trevisan e Seabra (2012). Na primeira etapa, a criança deve ligar os estímulos (cachorrinhos) em ordem crescente de tamanho. $\mathrm{Na}$ etapa seguinte, há dois estímulos diferentes (cachorros e ossos) e a criança deve ligá-los, alternadamente, seguindo a ordem de tamanho. Antes de iniciar o teste, a criança realiza o treino. Todo o tempo usado para conclusão de cada etapa, bem como para o traçado da criança (número de itens corretamente ligados), é registrado e contabilizado. A tarefa é interrompida quando a criança ligar todos os itens da tela (de forma correta ou incorreta) ou pelo examinador por meio do botão "avançar", se a criança não finalizar, por exemplo, ou se não tocar em todos os itens da tela.

\section{Flexibilidade Cognitiva para Crianças em Idade Escolar}

Igualmente ao teste elaborado para as crianças de 4 a 6 anos, o Teste de Flexibilidade para crianças em idade escolar é uma atividade adaptada, para uso em tablet, do Teste de trilhas: Parte A e B (Montiel \& Seabra, 2012). O teste é destinado às crianças em idade escolar $(7$ a 10 anos de idade) com conhecimento das ordens numérica e alfabética. Ele também possui duas partes: A e B. Para a execução de ambas as partes, a criança recebe as orientações de forma auditiva após o toque no botão "áudio". Na parte A, há duas telas. Na primeira, a criança é orientada a unir, por meio do toque na tela, as letras em ordem alfabética e, na segunda tela, os números em ordem crescente. $\mathrm{Na}$ parte $\mathrm{B}$, a criança é orientada para que alterne entre os números e as letras apresentadas, em uma mesma tela, em sequência crescente e alfabética. Assim, com as tarefas de pré-escolares, há as telas de treino e o desempenho é medido em termos de tempo de execução, número de sequência e conexões realizadas no primeiro minuto de execução. A finalização da tarefa também ocorre conforme a tarefa de pré-escolares. 


\section{Tarefa de Controle Inibitório}

Foram encontrados na literatura vários paradigmas relacionados às tarefas de controle inibitório. Os mais populares são os paradigmas de Stroop (Stroop, 1935), Go/No-Go (Donders, 1969), N-back (Gevins \& Cutillo, 1993) e Flanker (Eriksen \& Eriksen, 1974). Para avaliar o controle inibitório no TAFE, foram selecionadas as tarefas de Flanker para embasar o desenvolvimento das atividades.

No TAFE, os estímulos utilizados são as imagens de cinco pássaros apresentados em uma linha horizontal. Em cada apresentação, a criança é orientada a indicar, por meio do toque na tela e o mais rápido possível, a direção do pássaro do centro. Todos os pássaros são apresentados com o mesmo tamanho e a mesma cor, até que uma resposta seja emitida ou até três segundos decorridos após a apresentação do estímulo. São administradas 30 telas com imagens congruentes e 30 telas com imagens incongruentes, intercaladas de forma aleatória. Após as telas de treino, a criança inicia o teste. As respostas da criança são registradas e computadas, considerando: erros por omissão, erros e acertos congruentes e erros e acertos incongruentes.

\section{Resultados da Etapa 3 - Estudo Piloto}

O resultado do estudo piloto permitiu verificar, em uma pequena escala, o funcionamento, de modo geral, das tarefas. Foram avaliados positivamente critérios como a motivação e interesse das crianças para realizar as tarefas e a compreensão dos comandos e das etapas de treino. O tempo despendido para a realização de todas as tarefas também foi avaliado e, embora tenha variado conforme a faixa etária, constatou-se a possibilidade de realizar as quatros tarefas em um único momento.

\section{Resultados da Etapa 4 - A Análise dos Juízes}

Os questionários respondidos pelos juízes continham perguntas relacionadas aos conceitos dos construtos abordados nas tarefas, ao design das tarefas, ao layout geral do instrumento, ao cadastro do participante, à adequação das atividades para o público infantil, ao áudio das tarefas, à clareza das instruções fornecidas para as crianças, ao funcionamento das fases de treino, às regras de interrupção e finalização e, por fim, à correção e forma de registro dos resultados.
As respostas dos juízes foram analisadas de forma qualitativa, uma vez que as questões eram abertas e os juízes puderam escrever sugestões e orientações para contribuir na validade do teste. Todos os critérios foram avaliados como adequados. Contudo, foram sugeridas mudanças, como o acréscimo de dois itens no cadastro da criança (lateralidade e um campo para o registro das observações extras sobre o examinando), a adequação da linguagem (prosódia) da instrução fornecida na tarefa de memória de trabalho visual que estava demasiadamente mecanizada, segundo a avaliação dos juízes, e ainda a inclusão da possibilidade de encerrar a tarefa a qualquer momento e a possibilidade de retornar às instruções da tarefa quando necessário. Todas as mudanças sugeridas foram realizadas, uma vez que as alterações contribuíam para melhor desempenho e funcionamento das tarefas. Após essas modificações, concluiu-se a versão do instrumento e, então, foi conduzida a análise descrita a seguir.

\section{Resultados da Etapa 5 - Estudos Psicométricos Iniciais}

As principais medidas dos instrumentos foram analisadas a partir da correlação de Spearman e estão apresentadas na Tabela 1. As tarefas de memória de trabalho apresentaram correlações significativas (alta a moderada) com todas as outras medidas de memória de trabalho (verbal, visuoespacial e na ordem direta e inversa), com controle inibitório e com algumas medidas de flexibilidade cognitiva, sendo o maior número com as medidas de memória visuoespacial.

Entre as medidas de flexibilidade e inibição, foram encontradas correlações moderadas entre as medidas dos testes de flexibilidade para pré-escolares e as duas medidas do teste de inibição. Contudo, nos testes para escolares, a maioria das correlações com as tarefas de inibição foi baixa.

A fim de buscar evidências da fidedignidade do TAFE, foram realizadas correlações não paramétricas de Spearman entre as pontuações do TAFE aplicados em dois momentos diferentes. A Tabela 2 apresenta tais correlações. Das 25 medidas investigadas, 21 apresentaram valor de correlação significativa de magnitude classificada como muito alta, três apresentaram correlação alta e somente uma correlação está classificada como baixa.

Tabela 1

Análises de Correlação de Spearman entre as Principais Medidas no TAFE em Memória de Trabalho (MT), Flexibilidade Cognitiva (FC) e Controle Inibitório (CI)

\begin{tabular}{|c|c|c|c|c|c|c|c|c|c|c|c|}
\hline & & 1.1 & 1.2 & 1.3 & 1.4 & 1.5 & 1.6 & 1.7 & 1.8 & 1.9 & 2.0 \\
\hline \multirow[t]{2}{*}{ 1.2. MT visoespacial OI } & rho & 0,77 & & & & & & & & & \\
\hline & $p$ & $<0,001$ & & & & & & & & & \\
\hline \multirow[t]{2}{*}{ 1.3. MT verbal OD } & rho & 0,48 & 0,55 & & & & & & & & \\
\hline & $p$ & $<0,001$ & $<0,001$ & & & & & & & & \\
\hline
\end{tabular}


Tabela 1 (continuação)

Análises de Correlação de Spearman entre as Principais Medidas no TAFE em Memória de Trabalho (MT), Flexibilidade Cognitiva (FC) e Controle Inibitório (CI)

\begin{tabular}{|c|c|c|c|c|c|c|c|c|c|c|c|}
\hline & & 1.1 & 1.2 & 1.3 & 1.4 & 1.5 & 1.6 & 1.7 & 1.8 & 1.9 & 2.0 \\
\hline \multirow[t]{2}{*}{ 1.4. MT verbal OI } & rho & 0,52 & 0,66 & 0,59 & & & & & & & \\
\hline & $p$ & $<0,001$ & $<0,001$ & $<0,001$ & & & & & & & \\
\hline \multirow[t]{2}{*}{ 1.5. FC Pré-escolares A } & rho & 0,39 & 0,62 & 0,58 & 0,50 & & & & & & \\
\hline & $p$ & 0,054 & 0,001 & 0,003 & 0,012 & & & & & & \\
\hline \multirow[t]{2}{*}{ 1.6. FC Pré-escolares B } & rho & 0,53 & 0,44 & 0,26 & 0,38 & 0,37 & & & & & \\
\hline & $p$ & 0,008 & 0,03 & 0,209 & 0,061 & 0,079 & & & & & \\
\hline \multirow{2}{*}{$\begin{array}{l}\text { 1.7. FC escolares } \\
\text { letras sequências }\end{array}$} & rho & 0,30 & 0,40 & 0,33 & 0,06 & - & - & & & & \\
\hline & $p$ & 0,105 & 0,031 & 0,078 & 0,739 & - & - & & & & \\
\hline \multirow{2}{*}{$\begin{array}{l}\text { 1.8. FC escolares } \\
\text { número sequências }\end{array}$} & rho & 0,34 & 0,40 & 0,10 & 0,33 & - & - & 0,34 & & & \\
\hline & $p$ & 0,070 & 0,029 & 0,602 & 0,081 & - & - & 0,070 & & & \\
\hline \multirow{2}{*}{$\begin{array}{l}\text { 1.9. FC escolares } \\
\text { sequência parte } B\end{array}$} & rho & 0,48 & 0,41 & 0,03 & 0,12 & - & - & 0,49 & 0,44 & & \\
\hline & $p$ & 0,008 & 0,024 & 0,871 & 0,526 & - & - & 0,007 & 0,017 & & \\
\hline \multirow[t]{2}{*}{ 2.0 CI Incongruente } & rho & 0,59 & 0,67 & 0,33 & 0,50 & 0,37 & 0,51 & 0,31 & 0,41 & 0,36 & \\
\hline & $p$ & $<0,001$ & $<0,001$ & 0,017 & $<0,001$ & 0,07 & 0,011 & 0,091 & 0,026 & 0,049 & \\
\hline \multirow[t]{2}{*}{ 2.1 CI Congruente } & rho & 0,58 & 0,68 & 0,41 & 0,58 & 0,58 & 0,51 & 0,37 & 0,39 & 0,35 & 0,67 \\
\hline & $p$ & $<0,001$ & $<0,001$ & 0,002 & 0,002 & 0,002 & 0,011 & 0,043 & 0,032 & 0,056 & $<0,001$ \\
\hline
\end{tabular}

Tabela 2

Análises de Correlação de Spearman entre as Medidas no TAFE em Memória de Trabalho (MT), Flexibilidade Cognitiva (FC) e Controle Inibitório (CI) nas Duas Avaliações de Teste e Reteste

\begin{tabular}{|c|c|c|c|}
\hline Tarefa & rho & $p$ & $\mathrm{~N}$ \\
\hline MT Visoespacial - Ordem Direta & 0,94 & $<0,001$ & 10 \\
\hline MT Visoespacial - Ordem -Inversa & 0,91 & $<0,001$ & 10 \\
\hline MT Verbal - Ordem Direta & 0.99 & $<0,001$ & 10 \\
\hline MT Verbal - inv- Ordem Inversa ersa & 0,93 & $<0,001$ & 10 \\
\hline FC Pré-escolar parte A conexão & 1,00 & $<0,001$ & 4 \\
\hline FC Pré-escolar parte A sequência & 0,94 & 0,057 & 4 \\
\hline FC Pré-escolar parte A tempo & 1,00 & $<0,001$ & 4 \\
\hline FC Pré-escolar parte B conexão & 1,00 & $<0,001$ & 4 \\
\hline FC Pré-escolar parte B sequência & 1,00 & $<0,001$ & 4 \\
\hline FC Pré-escolar parte B tempo & 0,63 & 0,368 & 4 \\
\hline Escolar Letras - conexão & 1,00 & $<0,001$ & 6 \\
\hline FC Escolar Letras - sequência & 1,00 & $<0,001$ & 6 \\
\hline FC Escolar Letras - tempo & 0,89 & 0,015 & 6 \\
\hline FC Escolar número - conexão & 1,00 & $<0,001$ & 6 \\
\hline FC Escolar número - sequência & 1,00 & $<0,001$ & 6 \\
\hline FC Escolar número - tempo & 0,92 & 0,008 & 6 \\
\hline FC Escolar parte B - conexão & 1,00 & $<0,001$ & 6 \\
\hline FC Escolar parte B - sequência & 1,00 & $<0,001$ & 6 \\
\hline FC Escolar parte B - tempo & 0,77 & 0,070 & 6 \\
\hline FC Escolar soma parte A & 1,00 & $<0,001$ & 6 \\
\hline FC Escolar diferença entre B e A & 1,00 & $<0,001$ & 6 \\
\hline CI Inibição correta congruente & 0,68 & 0,031 & 10 \\
\hline CI Inibição correta incongruente & 0,91 & $<0,001$ & 10 \\
\hline CI Inibição incongruente - Congruente & 0,81 & 0,004 & 10 \\
\hline CI Erro omissão & 0,38 & 0,272 & 10 \\
\hline
\end{tabular}




\section{Discussão}

A iniciativa para o desenvolvimento do Teste Informatizado das Funções Executivas surgiu a partir das evidências de que as habilidades das FE são relevantes para diferentes situações da vida, da aprendizagem escolar e a manutenção de relacionamentos saudáveis (Diamond, 2013). Assim como os déficits no desenvolvimento dessas habilidades podem conduzir para dificuldades em controlar os impulsos, agir de forma reflexível e organizada, dificuldades em realizar julgamentos e tomar decisões. Adicionado a isso, há os achados da neurociência sobre as rápidas e robustas mudanças no desenvolvimento das FE no período da infância, sendo um período de acentuada neuroplasticidade (Zelazo \& Carlson, 2012). Tal contexto revela a importância de se ter instrumentos psicometricamente adequados para avaliar FE em crianças.

A escolha por um instrumento informatizado ocorreu visto que esse tipo de oferece padronização na aplicação, uma vez que mudanças sutis na apresentação da tarefa podem levar a resultados diferentes (Burgess \& Stuss, 2017). Adicionalmente, tal avaliação possibilita ao aplicador realizar observações de comportamentos paralelamente à testagem; oferece maior precisão nas medidas, inclusive nas de tempo; facilita a aplicação, permitindo ser administrado por profissionais com atuação em áreas distintas, como as de educação e saúde; e ainda permite o armazenamento de grande volume de dados coletados (Parsons, 2016).

A etapa de criação do instrumento possibilitou a escolha de tarefas simples com menor demanda de outras habilidades, uma vez que, dada a complexidade das $\mathrm{FE}$, outras diferentes demandas não executivas podem estar presentes em um teste de desempenho de FE. Tal problema, considerado um problema de impureza, é recorrente na prática clínica e muito citado na literatura (Strauss et al., 2006) e pode impactar na interpretação do desempenho da tarefa. Logo, disponibilizar um instrumento com menor interferência de outra habilidade para a prática clínica pode atenuar esse tipo problema. Outra forma de contornar tal dificuldade na clínica é o uso de técnicas estáticas, como a análise fatorial confirmatória e a modelagem de equação estrutural, embora pareçam pouco viáveis na prática clínica (Dias \& Malloy-Diniz, 2020), sendo mais acessível a criação de tarefas mais específicas para a habilidade-alvo. A criação das tarefas também proporcionou a viabilidade de se ter, para uso clínico, tarefas de desempenho com linguagem e design adequados para o público infantil. Essa característica é importante, visto que o processo de avalição neuropsicológica é usualmente longo, envolve outras tarefas e grande parte das crianças que se submete a esse processo apresenta queixas relacionadas ao desempenho cognitivo. Sendo assim, o processo de avaliação costuma ser pouco motivador e, por vezes, exaustivo, o que justifica uma maior facilidade quando as atividades possuem caráter lúdico.

Após o desenvolvimento das tarefas, o estudo piloto proporcionou a verificação, em contexto real, da compreensibilidade das instruções e, ainda, possibilitou observar o interesse das crianças pelas atividades. As etapas dedicadas aos estudos de validade e fidedignidade foram fundamentais, uma vez que um instrumento para ser considerado psicometricamente adequado deve apresentar evidências de validade e precisão (Pasquali, 2003). No presente estudo, a busca por evidências foi inicialmente conduzida com base no conteúdo, a partir da avaliação dos juízes, etapa fundamental na construção de um instrumento (Noronha et al., 2003). Verificou-se que o instrumento representou adequadamente os construtos teóricos que se objetivou avaliar, garantindo que os dados interpretados fossem os construtos almejados, sendo, assim, possível dar continuidade aos estudos de validade.

Para investigar as evidências baseadas na estrutura interna do instrumento, foram utilizadas análises de correlação de Spearman. Os dados revelaram diversas correlações significativas. Estas foram de magnitudes moderadas a altas, principalmente com as medidas de memória de trabalho; enquanto as de flexibilidade cognitiva foram as que apresentaram as menores magnitudes de correlação com as demais. Esses resultados remetem ao modelo de compreensão das FE proposto por Miyake et al. (2000) e revisado por Diamond (2013). Nesse modelo, os três componentes das funções são independentes, porém relacionados entre si, apoiando a ideia de unidade e diversidade das FE.

De fato, observou-se no presente estudo que a maioria das medidas apresentou correlação significativa entre si. Embora esse modelo de três componentes básicos tenha sido proposto a partir de uma amostra de universitários, quando se refere às crianças, o padrão mais comum encontrado foi o unitário, com evidências de que a diferenciação ocorre ao longo do desenvolvimento (Huizinga et al., 2006; St Clair-Thompson \& Gathercole, 2006). Esse padrão também é sustentado pelo estudo de Brydges et al. (2014), que identificou uma estrutura fatorial unitária em crianças entre 8 e 9 anos, porém uma estrutura bifatorial nas mesmas crianças avaliadas entre 10 e 11 anos de idade. Sendo que, no modelo bifatorial, o primeiro componente identificado foi a memória de trabalho, e o segundo integrou as habilidades de inibição e flexibilidade. Evidências também sustentadas por estudos de neuroimagem demonstram que, no início da infância, as regiões encefálicas ativadas durante uma tarefa de FE são mais amplas quando comparadas a outras crianças no final desse período (Fiske \& Holmaboe, 2019). No presente estudo, observou-se que, de fato, as habilidades apresentaram-se, na maioria dos casos, relacionadas entre si. Porém, frequentemente, com magnitudes moderadas, o que corrobora a hipótese de relação, mas não completa sobreposição entre elas. 
Ainda sobre a correlação encontrada entre as medidas, Diamond (2013) postula uma hierarquia na qual a memória de trabalho e a inibição contribuem para o desenvolvimento do terceiro componente básico, a flexibilidade cognitiva. Os dados do presente estudo adequam-se a essa hipótese da autora, uma vez que a memória de trabalho e a inibição apresentaram maior número e maior magnitude de correlação entre si, quando comparadas às medidas de flexibilidade. No presente estudo, não foi possível analisar separadamente as faixas etárias para buscar o número de componentes em cada fase, por exemplo, por meio de análises fatoriais. Sugere-se que estudos futuros, com maior número de participantes, possam conduzir tal análise, o que seria relevante para a compreensão das mudanças na estrutura das FE ao longo do desenvolvimento.

A investigação da precisão do instrumento foi obtida nos resultados entre as correlações do teste-reteste do TAFE. Correlações significativas, de magnitude moderada ou alta, foram encontradas na maioria das medidas, mostrando estabilidade dos escores ao longo do tempo. O tempo entre as duas avaliações (de 3 meses) pode ser questionado quando se refere à avaliação das FE, uma vez que, diferentemente de outras habilidades cognitivas, as FE apresentem maior flutuação ao longo do tempo (Burgess \& Stuss, 2017). Contudo, no presente estudo, evitou-se prolongar muito o tempo para além dos 3 meses, a fim de evitar a interferência de fatores ligados ao desenvolvimento e à aprendizagem dos participantes. No delineamento aqui usado, observou-se boa precisão do TAFE. No entanto, outras formas de analisar a precisão podem ser investigadas em estudos ulteriores.

\section{Considerações Finais}

O desenvolvimento das tarefas e os estudos preliminares das características psicométricas do instrumento TAFE demonstraram a viabilidade da utilização desse instrumento para a avaliação das FE no contexto brasileiro. No entanto, faz-se necessária a continuidade dos estudos psicométricos. Como limitações do estudo, os resultados foram analisados apenas de dois tipos de evidências de validade. Destacamos que os trabalhos seguintes devem complementar e investigar outros tipos de evidências, tais como relação com a idade, relações com outros instrumentos já validados de FE e comparação do desempenho entre amostras clínicas com queixas e diagnósticos que indicam prejuízos nas FE. É fundamental, ainda, aumentar e diversificar a amostra para a condução, por exemplo, de análises fatoriais e, futuramente, conduzir estudos de normatização para disponibilizar o instrumento ao uso clínico.

Apesar das limitações, ressalta-se que o desenvolvimento criterioso dessa ferramenta de investigação das FE em crianças deve contribuir para suprir, pelo menos parcialmente, a escassez de ferramentas nacionais padronizadas de avaliação das FE nessa faixa etária.

\section{Agradecimentos}

Não há menções.

\section{Financiamento}

Instituto Presbiteriano Mackenzie.

\section{Contribuições dos autores}

Declaramos que todos os autores participaram da elaboração do manuscrito. Especificamente, as autoras Glauce Karine Conti de Freitas Elage e Alessandra Gotuzo Seabra participaram da redação inicial do estudo - conceitualização, investigação, visualização, da análise dos dados e da redação final do trabalho - revisão e edição.

\section{Disponibilidade dos dados e materiais}

Todos os dados e sintaxes gerados e analisados durante esta pesquisa serão tratados com total sigilo devido às exigências do Comitê de Ética em Pesquisa em Seres Humanos. Porém, o conjunto de dados e sintaxes que apoiam as conclusões deste artigo estão disponíveis mediante razoável solicitação ao autor principal do estudo.

\section{Conflito de interesses}

Os autores declaram que não há conflitos de interesses.

\section{Referências}

Baddeley, A. (2000). The episodic buffer: A new component of working memory? Trends in Cognitive Sciences, 4(11), 417- 423. http://dx.doi. org/10.1016/S1364-6613(00)01538-2

Baggetta, P., \& Alexander, P. (2016). Conceptualization and operationalization of executive function. Mind, Brain, and Education, 10, 10-33. http://dx.doi.org/10.1111/mbe. 12100

Barkley, R. A. (2011). Is executive functioning deficient in ADHD? It depends on your definitions and your measures. The ADHD Report, 19(4), 1-9. https://doi.org/10.1521/adhd.2011.19.4.1

Bausela, H., Ustárroz, J., \& Cordero-Andrés, P. (2019). Déficits ejecutivos y trastornos del neurodesarrollo en la infancia y en la adolescencia. Revista de Neurología, 69(11), 461-469. https://doi.org/10.33588/rn.6911.2019133 
Best, J. R., Miller, P. H., \& Naglieri, J. A. (2011). Relations between executive function and academic achievement from ages 5 to 17 in a large, representative national sample. Learning and Individual Differences, 21(4), 327-336. https://doi.org/10.1016/j.lindif.2011.01.007

Bierman, K. L., \& Torres, M. (2016). Promoting the development of executive functions through early education and prevention programs. Em J. A. Griffin, P. McCardle \& L. S. Freund (Eds.), Executive function in preschool-age children: Integrating measurement, neurodevelopment, and translational research (pp. 299-326). Washington, DC, US: American Psychological Association. https://doi.org/10.1037/14797-014

Blankenship, T. L., Slough, M. A., Calkins, S. D., Deater-Deckard, K., Kim-Spoon, J., \& Bell, M. A. (2019). Attention and executive functioning in infancy: Links to childhood executive function and reading achievement. Developmental Science, 22(6), e12824. https:// doi.org/10.1111/desc. 12824

Brydges, C. R., Fox, A. M., Reid, C. L., \& Anderson, M. (2014). The differentiation of executive functions in middle and late childhood: A longitudinal latent-variable analysis. Intelligence, 47, 34-43. https://doi.org/10.1016/j.intell.2014.08.010

Burgess, P., \& Stuss, D. (2017). Fifty years of prefrontal cortex research: Impact on assessment. Journal of the International Neuropsychological Society, 23(9-10), 755-767. https://doi.org/10.1017/S1355617717000704

Carreiro, L. R. R., Dias, N. M., Malloy-Diniz, L. F., Trevisan, B. T., Minervino, C. A. S. M., Roazzi, A., \& Seabra, A. G. (2014). Testes de funções executivas no Brasil. Em A. G. Seabra, J. A. Laros, E. C. Macedo \& N. Abreu (Eds.), Inteligência e funções executivas: avanços e desafios para a avaliação neuropsicológica (pp. 84-111). São Paulo: Memnon.

Center on the Developing Child at Harvard University (2001). Building the brain's "Air trafic control" system: How early experiences shape the development of executive function. Working Paper, 11. Recuperado de http://www.developing child.harvard.edu

Christ, S., Stichter, J., O’Connor, K., Bodner, K., Moffitt, A., \& Herzog, M. (2017). Social skills intervention participation and associated improvements in executive function performance. Autism Research and Treatment, 5843851. https://doi.org/10.1155/2017/5843851

Corsi, P. M. (1972). Human memory and the medial temporal region of the brain. Dissertation Abstracts International, 34, 819B.

Dawson, P., \& Guare, R. (2018). Executive skills in children and adolescents: A practical guide to assessment and intervention. New York: Guilford Press.

Diamond, A. (2001). Prefrontal cortex development and development of cognitive functions. Em N. J. Smelser \& P. B. Baltes (Eds.), International Encyclopedia of the Social and Behavioral Sciences (pp. 11976-11982). Oxford: Pergamon.

Diamond, A. (2013). Executive functions. Annual Reviews of Psychology, 64, 135-168. https://doi.org/10.1146/annurev-psych-113011-143750.

Dias, N., \& Malloy-Diniz, L. (2020). Funções executivas: Modelos e aplicações. São Paulo: Pearson.

Dias, N., Menezes, A., \& Seabra, A. G. (2013). Age differences in executive functions within a sample of Brazilian children and adolescents. The Spanish Journal of Psychology, 16, E9, 1-14. https://doi.org/10.1017.sip.2013.12

Donders, F. C. (1969). On the speed of mental processes. Acta Psychologic, 30, 412-431.

Duckworth, A. L., Taxer, J. L., Eskreis-Winkler, L., Galla, B. M., \& Gross, J. J. (2019). Self-control and academic achievement. Annual Review of Psychology, 70, 373-399. https://doi.org/10.1146/annurev-psych-010418-103230

Eriksen, B. A., \& Eriksen, C. W. (1974). Effects of noise letters upon the identification of a target letter in a no search task. Perception $\mathcal{E}$ Psychophysics, 16(1), 143-149. https://doi.org/10.3758/BF03203267

Farrell-Pagulayan, K., Busch, R. M., Medina, K. L., Bartok, J.,A., \& Krikorian, R. (2006). Developmental normative data for the Corsi Block-tapping task. Journal of Clinical and Experimental Neuropsychology, 28(6), 1043-52. https://doi.org/10.1080/13803390500350977

Figueiredo, V. L. M., \& Nascimento, E. (2007). Desempenho nas duas tarefas do subteste Dígitos do WISC - III e do WAIS - III. Psicologia: Teoria e Pesquisa, 23(3), 313-138. https://doi.org/10.1590/S0102-37722007000300010

Fiske, A, \& Holmboe, K. (2019). Neural substrates of early executive function development. Developmental Review, 52, 42-62. https://doi. org/10.1016/j.dr.2019.100866

Fonseca, R. P., Prando, M. L., \& Zimmermann, N. (2016). Tarefas para avaliação Neuropsicológica - Avaliação de linguagem e funções executivas em crianças. São Paulo: Memnon.

Gevins, A. S., \& Cutillo, B. C. (1993). Neuroelectric evidence for distributed processing in human working memory. Electroencephalography and Clinical Neurophysiology, 87(3), 128-143.

Huizinga, M., Dolan, C. \& van der Molen, M. (2006). Age-related change in executive function: Developmental trends and a latent variable analysis. Neuropsychologia, 44(11), 2017-36. https://doi.org/10.1016/j.neuropsychologia

International Test Commission (2010). Guidelines for translating and adapting tests. Recuperado de http://www.intestcom.org

Johansson, M., Forssman, L., \& Bohlin, G. (2014). Individual differences in 10-month-olds' performance on the A-not-B task. Scandinavian Journal of Psychology, 55(2), 130-135. https://doi.org/10.1111/sjop.12109

Joly, M. C. R. A. Welter, G. M. R., Martins, R. X., Marini, Montiel, J. M., Lopes, F., \& Carvalho, M. R. (2005). Sistema de avaliação para testes informatizados (SAPI): Estudos preliminares. Revista de Psicologia da Vetor Editora, 6(2), 51-60.

Malloy-Diniz, L., Timóteo, A., Serpa, A., \& Querino, E. (2020). EF Solutions [Web page]. Recuperado de https://metacognitiv.com/ef-solutions

Mata, F., Sallum, I., Moraes, P. H. P. D., Miranda, D. M., \& Malloy-Diniz, L. F. (2013). Development of a computerized version of the Children's Gambling Task for the evaluation of affective decision-making in Brazilian preschool children. Estudos de Psicologia (Natal), 18(1), 151-157. https://doi.org/10.1590/S1413-294X2013000100024

McClelland, M. M., Cameron, C. E., Duncan, R., Bowles, R. P., Acock, A. C., Miao, A., \& Pratt, M. E. (2014). Predictors of early growth in academic achievement: the head-toes-knees-shoulders task. Frontiers in Ppsychology, 5, 1-14. https://doi.org/10.3389/fpsyg.2014.00599

Merchán-Naranjo, J., Boada, L., Del Rey-Mejías, A., Mayoral, M., Llorente, C., Arango, C., \& Parellada, M. (2016). Executive function is affected in autism spectrum disorder, but does not correlate with intelligence. Revista de Psiquiatría y Salud Mental, 9(1), 39-50. https:// doi.org/10.1016/j.rpsm.2015.10.005

Miyake, A., \& Friedman, N. P. (2012). The nature and organization of individual differences in executive functions: Four general conclusions. Current Directions in Psychological Science, 21(1), 8-14. https://doi.org/10.1177/0963721411429458

Miyake, A., Friedman, N. P., Emerson M.J., Witzki, A. H., \& Howerter, A. (2000). The unity and diversity of executive functions and their contributions to complex "Frontal lobe" tasks: A latent variable analysis. Cognitive Psychology, 41(1), 49-100. https://doi.org/10.1006/cogp.1999.0734

Montiel, J. M., \& Seabra, A. G. (2012). Teste de Atenção por Cancelamento. Em A. G. Seabra, N. M. Dias (Eds.), Avaliação neuropsicológica cognitiva: Atenção e funções executivas (pp. 57-66). São Paulo: Memnon.

Noronha, A. P., Vendramini, C. M., Canguçu, C., Souza, C. V., Cobêro, C., Paula, L., Franco, M., Lima, O. M., Guerra, P. B., \& Filizatti, R. (2003). Propriedades psicométricas em manuais de testes de inteligência. Psicologia em Estudo, 8(1), 93-99. https://doi.org/10.1590/ S1413-73722003000100012 
Olea, J., Abad, F. J., \& Barrada, J. R., (2010). Tests informatizados y otros nuevos tipos de tests. Papeles del Psicólogo, 31(1), 94-107. https:// doi.org/10.1017/ CBO9781107415324.004

Oliveira, M. S. \& Rigoni, M. S. (2010). Figuras Complexas de Rey: Teste de Cópia e de Reprodução de Memória de Figuras Geométricas Complexas. São Paulo, SP: Casa do Psicólogo.

Parsons, T. D. (2016). Neuropsychological assessment 2.0: Computer automated assessments. Em T. D. Parsons (Ed.), Clinical Neuropsychology and Technology (pp. 47-63). Cham: Springer.

Partington, J. E., \& Leiter, R. G. (1949). Partington's Pathways Test. Psychological Service Center Bulletin, 1, 9-20.

Pasquali, L. (2003). Psicometria: Teoria dos testes na psicologia e na educação. Rio de Janeiro: Vozes.

Primi, R. (2010). Avaliação psicológica no Brasil: Fundamentos, situação atual e direções para o futuro. Psicologia: Teoria e Pesquisa, 26(n/e), 25-38. https://doi.org/10.1590/S0102-37722010000500003

Rothbart, M. K., Sheese, B. E., Rueda, M. R., \& Posner, M. I. (2011). Developing mechanisms of self-regulation in early life. Emotion Review, 3(2), 207-213. https://doi.org/10.1177/1754073910387943

Rueda, F. J. M.; Noronha, A. P. P., Sisto, F. F., Santos, A. A. A., \& Castro, N. R. (2013). Escala Wechsler de Inteligência para Crianças - WISC-IV. São Paulo, SP: Casa do Psicólogo.

Santos, F. H., \& Bueno, O. F. A. (2003). Validation of the Brazilian children's test of pseudoword repetition in Portuguese speakers aged 4 to 10 years. Brazilian Journal of Medical and Biological Research, 36(11), 1633-1547. https://doi.org/10.1590/S0100-879X2003001100012

Santos, F. H., \& Engel de Abreu, P. (2009). Adaptação Brasileira da AWMA - "Automated Working Memory Assessment". Em K. Z. Ortiz, L. D. Mendonça, A. Foz, C. D. Santos, D. Fuentes \& D. D. Azambuja (Eds.), Avaliação Neuropsicológica: Panorama interdisciplinar dos estudos atuais na normatização e validação de instrumentos no Brasil (pp. 355-378). São Paulo: Vetor.

Santos, F. H., Mello, C. B., Bueno, O. F. A., \& Dellatolas, G. (2005). Cross-cultural differences for three visual memory tasks in Brazilian children. Perceptual and Motor Skills, 101(2), 421-433. https://doi.org/10.2466/pms.101.2.421-433

Seabra, A. G., Dias, N. M., \& Bosa, C. (2017). Funções executivas: Definição do construto, principais alterações em TEA e instrumentos de avaliação no contexto nacional. Em C. Bosa \& M. C. T. V. Teixeira (Eds.), Autismo: Avaliação psicológica e neuropsicológica (pp. 179-203). São Paulo. Hogrefe.

St Clair-Thompson, H. L., \& Gathercole, S. E. (2006). Executive functions and achievements in school: Shifting, updating, inhibition, and working memory. Quarterly Journal of Experimental Psychology, 59(4), 745-759. https://doi.org/10.1080/17470210500162854

Strauss, E., Sherman, E. M., \& Spreen, O. (2006). A compendium of neuropsychological tests: Administration, norms, and commentary. New York: Oxford University Press.

Stroop, J. R. (1935). Studies of interference in serial verbal reactions. Journal of Experimental Psychology, 18(6), 643-662.

Trevisan, B. T., \& Seabra, A. G. (2012). Teste de trilhas para pré-escolares. Em A. G. Seabra, \& N. M. Dias (Eds.), Avaliação neuropsicológica cognitiva: Atenção e funções executivas (pp. 92-100). São Paulo: Memnon.

Uehara, E., Mograbi, D., Charchat-Fichman, H., \& Fernandez, J. L. (2016). Evidências de validade de um instrumento executivo informatizado infantil: Jogo das Cartas Mágicas. Revista Neuropsicologia Latinoamericana, 8(1), 22-34.

Urbina, S. (2007). Fundamentos da testagem psicológica. Porto Alegre: Artmed.

Vandierendonk, A., Kemps, E., Fastame, M. C., \& Szmalec, A. (2004) Working memory components of the Corsi blocks task. British Journal of Psychology, 95(1), 57-79. https://doi.org/10.1348/000712604322779460

Williams, P. E., Lawrence, W. G., \& Rolfhus, E. L. (2003). Psychometric properties. (WISC-IV Technical Report no. 2). San Antonio: Psychological Corp.

Wolf, S., \& McCoy, D. (2019). The role of executive function and social-emotional skills in the development of literacy and numeracy during preschool: A cross-lagged longitudinal study. Developmental Science, 22(4), e12800. https://doi.org/10.1111/desc.12800

Zelazo, P. D., \& Carlson, S. M. (2012). Hot and cool executive function in childhood and adolescence: Development and plasticity. Child Development Perspectives, 6(4), 354-360. https://doi.org/10.1111/j.1750-8606.2012.00246.x

Zelazo, P. D., Müller, U., Frye, D., \& Marcovitch, S. (2003). The development of executive function in early childhood: I. The development of executive function. Monographs of the Society for Research in Child Development, 68(3), 11-27. https://doi.org/10.1111/j.0037976X.2003.00261.x

\section{Sobre as autoras}

Glauce Karine Conti de Freitas Elage é psicóloga, Mestre e Doutoranda em Distúrbios do Desenvolvimento pela Universidade Presbiteriana Mackenzie, São Paulo, SP. Docente no programa de Pós-Graduação lato Sensu em Neuropsicologia da Pontifícia Universidade Católica de Goiás.

Alessandra Gotuzo Seabra é psicóloga, Doutora em psicologia pela USP. Docente do Programa Universidade Presbiteriana Mackenzie, São Paulo, SP, Bolsista de Produtividade do CNPq.

\section{Como citar este artigo}

Elage, G. K. C. F., \& Seabra, A. G. (2021). Desenvolvimento e Propriedades Psicométricas do Teste Informatizado de Avaliação das Funções Executivas. Avaliação Psicológica, 20(1), 100-110. http://dx.doi.org/10.15689/ap. 2021.2001.17491.11 